\title{
DNA replication defect in the Escherichia coli cgtA(ts) mutant arising from reduced DnaA levels
}

\begin{abstract}
In Escherichia coli and other bacteria, the ribosome-associated CgtA GTP-binding protein plays a critical role in many basic cellular processes, including the control of DNA replication and/or segregation. However, the mechanism of this control is largely unknown. Here we report that ectopic expression of the dnaA gene partially restored both early growth in liquid medium and DNA synthesis defects of the cgtA(ts) mutant. Amounts of DnaA protein in the $\operatorname{cgtA}(\mathrm{ts}) \mathrm{mu}-$ tant incubated at elevated $\left(42^{\circ} \mathrm{C}\right)$ temperature were significantly lower relative to wild-type bacteria. Both level of $d n a A$ mRNA and transcriptional activity of the dnaA promoter-lac $Z$ fusion were decreased in the CgtA-deficient cells. The effects of ectopic expression of $d n a A$ were specific as analogous expression of another gene coding for a replication regulator, seqA, had no significant changes in growth and DNA synthesis in the cgtA mutant. Thus, it appears that the DNA replication defect in this mutant is a consequence of reduced DnaA levels.
\end{abstract}

Keywords Obg proteins - CgtA protein - GTP-binding proteins - DNA replication - DnaA initiator protein . SeqA protein
A. E. Sikora $\cdot$ R. Zielke · G. Węgrzyn $(\bowtie)$

Department of Molecular Biology, University of Gdansk, Kładki 24, 80-822, Gdansk, Poland

E-mail: wegrzyn@biotech.univ.gda.pl

Tel.: + 48-58-3463014

Fax: + 48-58-3010072

\section{A. E. Sikora $\cdot$ R. Zielke}

Department of Molecular, Cellular and Developmental Biology, University of Michigan, Ann Arbor, MI 48109-1048, USA

\section{A. Węgrzyn}

Laboratory of Molecular Biology (affiliated with the University of Gdańsk), Institute of Biochemistry and Biophysics,

Polish Academy of Sciences, Kładki 24, 80-822, Gdansk, Poland

\section{Introduction}

GTP-binding proteins have been found in all living organisms examined thus far, and play crucial roles in the regulation of fundamental cellular processes. One subfamily of the evolutionarily conserved GTP-binding proteins consists of the bacterial Obg proteins (for reviews see Caldon et al. 2001; Caldon and March 2003; Czyż and Węgrzyn 2005). This subfamily, together with four other subfamilies (DRG, YyaF/YchF, Ygr210 and NOG1) forms the OBG family, which includes archeal-, bacterial- and eukaryotic-GTPbinding proteins (Leipe et al. 2002). The essential nature of Obg proteins in Bacillus subtilis (Trach and Hoch 1989), Streptomyces griseus (Okamoto et al. 1997), Streptomyces coelicolor (Okamoto and Ochi 1998), Caulobacter crescentus (Maddock et al. 1997), Escherichia coli (Arigoni et al. 1998; Kobayashi et al. 2001), and yeast ortholog Noglp (Park et al. 2001), assumed to be due to their requirement for ribosomal functions and DNA transactions, confirms the importance of the role of OBG family.

Trach and Hoch (1989) discovered a gene coding for a member of the $\mathrm{Obg} / \mathrm{GTP} 1$ subfamily in $B$. subtilis. Its name is ' $\mathrm{SpoOB}$-associated GTP-binding protein', since the $o b g$ gene is located immediately downstream of and co-transcribed with $\operatorname{spoOB}$, and $\mathrm{Obg}$ protein was found to be involved in the control of sporulation. It was proposed that Obg can function by sensing intracellular GTP level (Kok et al. 1994), and may be required to stimulate the activity of the phosphorelay system (Vidwans et al. 1995). More recent evidence points to a role of $\mathrm{Obg}$ in ribosome function. In B. subtilis cells, the $\mathrm{Obg}$ protein exists as a large cytoplasmic complex, co-elutes with ribosomal subunits and specifically interacts with the 50S ribosomal subunit protein L13 (Scott et al. 2000). Similar to the Obg protein of B. subtilis, the CgtA proteins (homologues of $B$. subtilis obg gene product) of $C$. crescentus (Lin et al. 2004) and E. coli are associated with 50S ribosomal subunit and interact with some other 
proteins, for example, SpoT and DnaK (Sato et al. 2005; Wout et al. 2004). However, perhaps unexpectedly, recent studies on conditional $C$. crescentus cgt $A$ mutants revealed that the lethal phenotype of $\operatorname{cgt} A$ dysfunction is not due to impaired ribosome function (Datta et al. 2004).

As mentioned, the Obg-like proteins are involved in many crucial physiological processes, and are found to be essential proteins in many bacteria. However, despite determination of X-ray structures of $\mathrm{N}$-terminal 342 amino acids of $\mathrm{Obg}$ from $B$. subtilis (Buglino et al. 2002) and its full-length homolog from Thermus thermophilus (Kukimoto-Niino et al. 2004), their exact cellular functions are still largely unknown. Moreover, different conclusions about the involvement of Obg-like proteins in crucial processes were published. Perhaps the most intriguing example is the putative influence of $\mathrm{obg} / \mathrm{cgtA}$ gene functions on DNA replication. Kok et al. (1994) suggested that Obg may be involved in the control of DNA replication in B. subtilis, but the proposed hypothesis was highly speculative. On the other hand, Kobayashi et al. (2001), on the basis of flow cytometry analysis, concluded that the dysfunction of $\operatorname{cgt} A$ does not impair DNA synthesis in E. coli. They suggested that CgtA (ObgE) directly or indirectly participates in chromosome partitioning. Contrary to the conclusion presented by Kobayashi et al. (2001), Datta et al. (2004) proposed that CgtA is necessary for DNA replication, progression through the cell cycle and in 50S ribosomal subunit biogenesis in $C$. crescentus. Since the biochemical properties of CgtA proteins from $C$. crescentus and $E$. coli are very similar (Wout et al. 2004), the two hypotheses presented here are contradictory. Moreover, it was also demonstrated that replication of some, but not all, plasmid replicons is affected in E. coli cgtA mutant cells (Ulanowska et al. 2003). Very recently, Foti et al. (2005) proposed that CgtA is involved in the promotion of bacterial cell survival when replication forks are arrested. They have isolated an E. coli mutant in the $\operatorname{cgt} A$ gene (called $\operatorname{obg} E$ by them), which was very sensitive to various DNA-replication inhibitors. Genetic analysis suggested that chromosome breaks and regressed replication forks may accumulate in this mutant. Nevertheless, on the basis of results reported by Foti et al. (2005), one could not exclude that DNA replication initiation is affected in the absence of CgtA function (as noted by those authors and discussed by Michel 2005).

Because of contradictory conclusions about the potential role of CgtA in DNA replication, presented by different authors and mentioned previously, we aimed to investigate this problem and to answer the question whether $\operatorname{cgt} A$ gene function is involved in the regulation of DNA replication initiation in E. coli. In our studies, we used strains employed previously by Kobayashi et al. (2001), to avoid any possible confusion due to various phenotypes of different mutants. Our results indicate that CgtA influences DNA replication by regulating dnaA gene expression.

\section{Materials and methods}

Bacterial strains, plasmids and growth conditions

A pair of otherwise isogenic E. coli MG1655 AcgtA::kan mutants harboring $p_{\mathrm{BAD}}:: c g t A$ or $p_{\mathrm{BAD}}:: c g t A(\mathrm{ts})$ on pSC101-derived plasmids (GN5002 and GN5003, respectively) (Kobayashi et al. 2001), hereafter referred to as wild type and $\operatorname{cgtA}(\mathrm{ts})$ strains, were used throughout this study. DNA sequencing confirmed the presence of the mutated gene, whose product is a CgtA $(\mathrm{ObgE})$ protein carrying both $\mathrm{G} 80 \mathrm{E}$ and $\mathrm{D} 85 \mathrm{~N}$ substitutions (data not shown). Complementation of the $\operatorname{cgtA}$ mutant phenotype by expression of the wild-type allele from a plasmid is observed (Kobayashi et al. 2001; and data not shown). Bacterial cells were grown at $30^{\circ} \mathrm{C}$ in Luria-Bertani liquid medium (LB: Sambrook et al. 1989) with shaking or on LB agar plates (LB with $1.5 \%$ bactoagar), unless otherwise indicated. The medium was supplemented with kanamycin $\left(30 \mu \mathrm{g} \mathrm{ml}^{-1}\right)$ and chloramphenicol $\left(20 \mu \mathrm{g} \mathrm{ml}^{-1}\right)$, and arabinose was added to final concentrations of $0.001 \%$ for GN5002 or $0.1 \%$ for GN5003 to achieve protein levels in these strains that were approximately equivalent to that seen in MG1655 (data not shown). Ampicillin $\left(50 \mu \mathrm{g} \mathrm{ml}^{-1}\right)$ was added when strains harbored the pdnaA116 plasmid (see next). For all experiments described in this report, overnight cultures were diluted 1:100, cultivated to an $\mathrm{OD}_{600}$ of 0.05 at $30^{\circ} \mathrm{C}$, then divided and grown at either 30 or $42^{\circ} \mathrm{C}$.

pdnaA116 is a ColE1-like replicon bearing the $p_{\text {lac }}{ }^{-}$ dnaA transcription fusion and $\mathrm{lacI}^{q}$ and bla genes (Krause et al. 1997). Plasmid pMAK7, containing the seq $A$ gene under the control of the $p_{\text {lac }}$ promoter, has been described earlier (von Freiesleben et al. 2000).

For construction of fusions of $d n a A$ and seqA promoter regions with lac $Z$, plasmid pHG86 (Giladi et al. 1992) was used as a vector. The dnaA promoter region (positions -489 to +47 ) was obtained by PCR using primers pdnaABam (5'-ATT CAG GAT CCT TGA CGT ACG TCG) and pdnaAEco (5'-CAC TCC GGA GTG AAT TCT CTT TCC). The seqA promoter region (positions -240 to +4 ) was obtained by PCR using primers pseqABam (5'-CAA TCG TTT TCA TCT GGA TCC AGT GC) and pseqAEco (5'-ATG GGA GAA TTC TTG TGC TGG TTT TG). The resultant PCR fragments, containing either the dna $A$ or the seq $A$ promoter region, were digested with Eco RI and BamHI, and inserted into the corresponding sites of the pHG86 vector.

Measurement of DNA and RNA synthesis

For the measurement of intracellular DNA or RNA synthesis, cells were grown at either 30 or $42^{\circ} \mathrm{C}$ until the cultures reached an $\mathrm{OD}_{600}$ of 0.2 prior to the addition of either $\left[{ }^{3} \mathrm{H}\right]$ thymidine or $\left[{ }^{3} \mathrm{H}\right]$ uridine (MP Biomedicals) to 
a final concentration of 10 or $2 \mu \mathrm{Ci} \mathrm{ml}^{-1}$, respectively. To induce the stringent response, DL-serine hydroxamate was added to $1 \mathrm{mg} \mathrm{ml}^{-1}$. Aliquots $(50 \mu \mathrm{l}$ each) were withdrawn at indicated times, placed onto Whatmann 3 paper filters $(2 \mathrm{~cm}$ diameter $)$ and then transferred immediately to ice-cold $10 \%$ trichloracetic acid (TCA). Following sequential washes $(5 \%$ TCA, 1\% TCA and twice in $96 \%$ ethanol), the filters were air-dried and radioactivity was measured in a scintillation counter (Beckman LS3133P).

\section{Estimation of DnaA protein level in cells}

Cells were grown, as described previously, and an equivalent cell masses were pelleted by centrifugation, lysed in sodium dodecyl sulfate (SDS) buffer and separated by SDS-polyacrylamide gel electrophoresis $(12 \%)$ (Sambrook et al. 1989). After electrophoretic separation and transfer to Protran membrane (Schleicher and Schuel, following manufacturer's recommendations), 1/ 500 anti-DnaA antibodies (kindly provided by Dr. Igor Konieczny, Intercollegiate Faculty of Biotechnology, University of Gdańsk and Medical University of Gdańsk) and 1/10,000 anti horseradish peroxidase-conjugated anti-rabbit antibodies were used for immunoblotting (Sambrook et al. 1989). The signal was detected by fluorography with ECL (Amersham Pharmacia Biotech). Purified DnaA protein, used as a standard, was kindly provided by Dr. Agnieszka Szalewska-Palasz (Department of Molecular Biology, University of Gdańsk).

\section{RNA dot-blot hybridization}

Total RNA was isolated from bacterial cell mass equal to two OD units using Total RNA Prep Plus kit (A\&A Biotechnology). Genomic DNA was removed by incubation with DNase (RNase-free, Roche). RNA was spotted onto Zeta Probe Blotting Membranes (BioRad), using Bio-DotApparatus (BioRad). Following baking for $2 \mathrm{~h}$ at $80^{\circ} \mathrm{C}$ in a hybridization oven (OV3, Biometra), membranes were washed for $2 \mathrm{~min}$ in $2 \times \operatorname{SSC}(0.3 \mathrm{M}$ $\mathrm{NaCl}, 0.03 \mathrm{M}$ sodium citrate). The prehybridization was performed for $2 \mathrm{~h}$ at $65^{\circ} \mathrm{C}$ in a hybridization buffer $[0.75 \mathrm{M} \mathrm{NaCl}, 0.075 \mathrm{M}$ sodium citrate, $0.05 \mathrm{M}$ phosphate-buffer, $\mathrm{pH} 6.5,0.5 \%$ Blocking reagent (NEN)] supplemented with $100 \mu \mathrm{g} \mathrm{ml}^{-1}$ of herring sperm DNA (Sigma). Dot blot hybridization was performed overnight at $65^{\circ} \mathrm{C}$ in a hybridization buffer supplemented with fluorescein-labeled probes designed for each specific transcript (for dnaA, dnaA1: 5'-AT CTT CTT TGA TAT CGT GGC TCT CTT CAC G; for seqA, seqA1, 5'-CGA TAT GCT TAG TGT GGC TGG CAA TAT AGC). After hybridization, membranes were washed twice for $15 \mathrm{~min}$ in a buffer composed of $15 \mathrm{mM} \mathrm{NaCl}$, $1.5 \mathrm{mM}$ sodium citrate, and $0.1 \% \mathrm{SDS}$, then $2 \mathrm{~min}$ in buffer A $(100-\mathrm{mM}$ Tris- $\mathrm{HCl}, 600 \mathrm{mM} \mathrm{NaCl})$ and addi- tional $30 \mathrm{~min}$ in buffer A supplemented with $0.5 \%$ Blocking reagent (NEN). Then, the membranes were incubated in buffer A supplemented with $0.5 \%$ BSA and Antifluorescein-AP conjugated antibodies (Perkin Elmer Life Science). Detection of reaction products was performed using CDP-Star Chemiluminescent Reagent (Perkin Elmer Life Science).

Measurement of $\beta$-galactosidase activity in cells

The $\beta$-galactosidase activities were determined in $E$. coli strain $\operatorname{cgt} A$ (ts) carrying $p_{\mathrm{dnaA}}-l a c Z$ or $p_{\mathrm{seqA}}$-lac $Z$ fusions according to Miller (1972).

Since multicopy gene fusions were used in these experiments, $\beta$-galactosidase activity was calculated per plasmid copy number. Plasmid copy number was estimated by the isolation of plasmid DNA (using DNA isolation kit, A\&A Biotechnology), EcoRI digestion, separation in $1 \%$ agarose gel during electrophoresis, and staining with ethidium bromide $\left(0.5 \mu \mathrm{g} \mathrm{ml}^{-1}\right)$. The relative amount of DNA in each band was estimated by densitometry, using Quantity One software (BioRad).

\section{Results}

Ectopic expression of dnaA partially suppresses the growth defect of the cgtA(ts) mutant

Previous studies demonstrated that the E. coli $\operatorname{cgt} A$ (ts) mutant strain GN5003 is deficient in the replication of some, but not all, types of plasmid replicons (Ulanowska et al. 2003). Furthermore, in preliminary experiments, it was indicated that in the $\operatorname{cgt} A$ (ts) mutant the levels of the DNA initiator protein, DnaA, were decreased (Ulanowska et al. 2003). DnaA, in addition to playing a role as an initiation-specific protein (Fuller et al. 1984), also functions as a transcriptional activator or a repressor of a number of genes (Messer and Weigel 2003). One possible explanation of those results, therefore, is that the DNA replication and cell division phenotype of the $\operatorname{cgt} A$ (ts) mutant is a consequence of a lower level of DnaA protein in the cell. To test this hypothesis, we examined the consequences of expressing dnaA, from a plasmid (pdnaA116) in the cgtA(ts) mutant.

We tested whether the introduction of additional copies of dnaA on plasmid pdnaA116 improved the growth defect of the $\operatorname{cgt} A(\mathrm{ts})$ mutant. Cultures of wild type or $\operatorname{cgtA}(\mathrm{ts})$ cells alone, or harboring pdnaA116, were monitored for growth at either 30 or $42^{\circ} \mathrm{C}$ (Fig. 1a). As expected, wild-type cells, irrespective of the presence of pdnaA116, grew at the same rate, although the growth of both wild-type strains was faster at $42^{\circ} \mathrm{C}$ than at $30^{\circ} \mathrm{C}$ (Fig. 1a). For the cgt $A$ (ts) mutant grown at either 30 or $42^{\circ} \mathrm{C}$, we observed similar slow growth rates during the first $3 \mathrm{~h}$. Continued incubation at $42^{\circ} \mathrm{C}$, however, resulted in a dramatic reduction in the growth of cells relative to those grown at $30^{\circ} \mathrm{C}$ (Fig. 1a), and a 


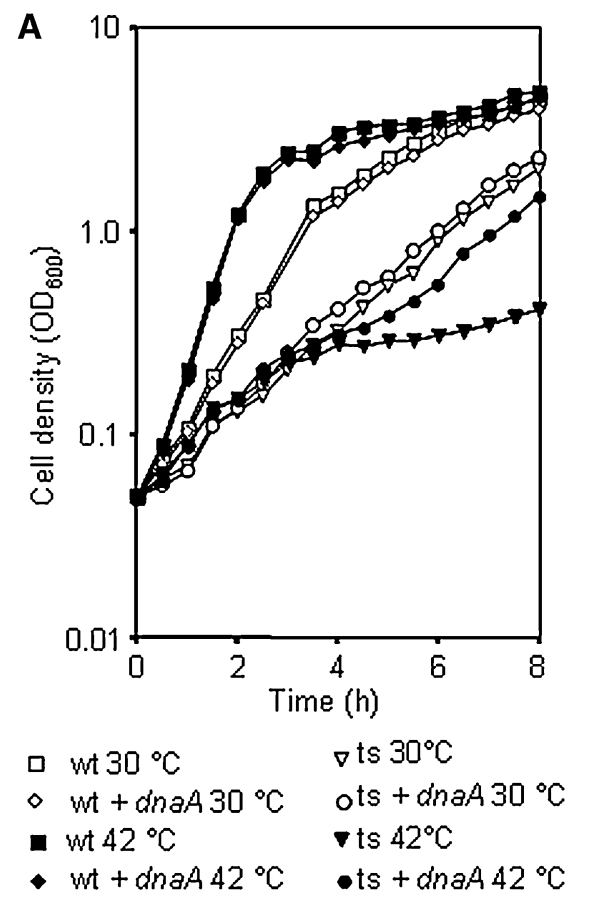

Fig. 1 Partial suppression of growth (a) and DNA replication (b) of the $\operatorname{cgt} A(\mathrm{ts})$ mutant harboring a plasmid-expressing dnaA. a Growth of wild-type and mutant cells harboring plasmid pdnaA116 (bearing the dnaA gene; $+d n a A$ ). The cell density of cultures of the indicated strains was monitored, over time, as indicated. Results obtained with wild-type cells $(w t)$ with (diamonds) or without (squares) pdnaA116 and cgtA(ts) mutants (ts) with (circles) and without (triangles) pdnaA116 are shown. Cells

significant number of elongated cells (Fig. 2), consistent with a previous report (Kobayashi et al. 2001). The addition of pdnaA116 to cgtA(ts) had no effect on mutant-cell growth at $30^{\circ} \mathrm{C}$, whereas growth at $42^{\circ} \mathrm{C}$ was improved, although not restored to the same levels seen in wild-type cells (Fig. 1a). Vast overproduction of DnaA, achieved by the induction of dnaA gene expression from pdnaA116 by 1-mM IPTG, did not improve the growth of the $\operatorname{cgt} A(\mathrm{ts})$ mutant at $42^{\circ} \mathrm{C}$, but this was apparently caused by a deleterious effect of a highly increased DnaA level on bacterial growth, observed in both $\operatorname{cgtA}{ }^{+}$and $\operatorname{cgt} A(\mathrm{ts})$ strains and at both 30 and $42^{\circ} \mathrm{C}$ (data not shown).

Contrary to the partial suppression of the growth defect of $\operatorname{cgt} A(\mathrm{ts})$ bacteria by pdnaA116, the morphology of these cells, was identical to that of the $\operatorname{cgt} A(\mathrm{ts})$ mutant cells grown at $42^{\circ} \mathrm{C}$ in the absence of the plasmid bearing the wild-type $d n a A$ allele (Fig. 2). Moreover, the cells were unable to form colonies on plates at the nonpermissive temperature (data not shown), indicating that long-term growth was not sustained. Thus, the ectopic expression of dnaA partially suppressed the growth defect of the $\operatorname{cgtA}(\mathrm{ts})$ mutant during early incubation times at the non-permissive temperature. When a culture of the $\operatorname{cgt} A$ (ts) mutant was incubated at $42^{\circ} \mathrm{C}$ for $6 \mathrm{~h}$, subsequent plating at $30^{\circ} \mathrm{C}$ revealed about tenfold decrease in the number of colony-forming units, relative to

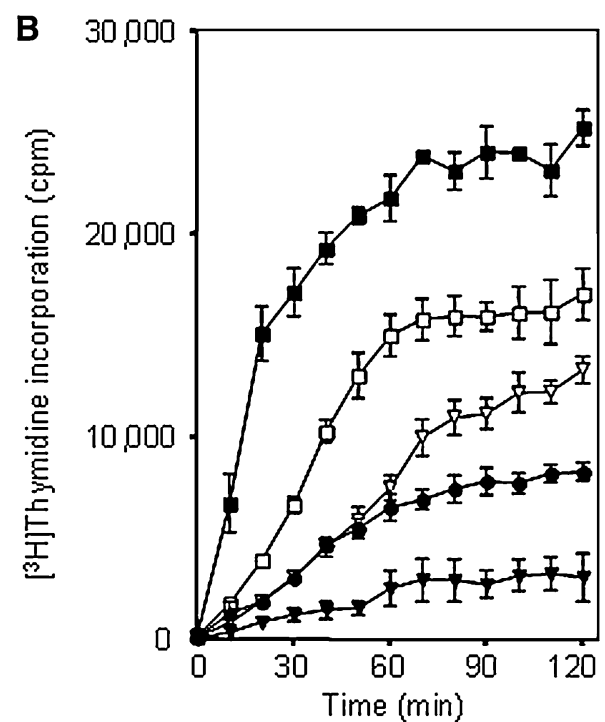

$$
\begin{array}{ll}
\text { - wt } 30^{\circ} \mathrm{C} & \nabla \text { ts } 30^{\circ} \mathrm{C} \\
\text { - wt } 42{ }^{\circ} \mathrm{C} & \text { rts } 42{ }^{\circ} \mathrm{C} \\
& \text { ots }+ \text { dna } 42{ }^{\circ} \mathrm{C}
\end{array}
$$

grown at $30^{\circ} \mathrm{C}$ are indicated by open symbols and cells shifted to $42^{\circ} \mathrm{C}$ are indicated by closed symbols. b Measurement of $\left[{ }^{3} \mathrm{H}\right]$ thymidine incorporation into DNA. Cells were grown as described in Materials and methods upon reaching an $\mathrm{OD}_{600}$ of 0.2 (labeled as $0 \mathrm{~min}$ ) before the addition of the radioactive DNA synthesis precursor. Shown are the results from three experiments \pm SD. Strain symbols are as in a

the same mutant but incubated in a liquid culture for $6 \mathrm{~h}$ at $30^{\circ} \mathrm{C}$. This mutant phenotype was completely restored (to the positive control level of the efficiency of plating) by the presence of pdnaA116 (data not shown).

Experiments analogous to those described in this subsection were performed with a plasmid expressing seq A, a gene coding for another regulator of DNA replication initiation. We found no significant effects of SeqA overproduction on the $\operatorname{cgt} A(\mathrm{ts})$ mutant growth (data not shown), indicating that the influence of ectopic expression of dnaA was specific.

DNA synthesis in $c g t A$ mutant cells expressing dnaA from a plasmid

To examine whether the rate of DNA synthesis was also suppressed in mutant strains harboring pdnaA116, we measured the incorporation of $\left[{ }^{3} \mathrm{H}\right]$ thymidine into cellular DNA (Fig. 1b). As expected, in the wild-type cells grown at either temperature, there was an initial increase in DNA synthesis followed by a reduction that mimicked the cell growth: cells in exponential growth incorporated more $\left[{ }^{3} \mathrm{H}\right]$ thymidine than cells entering stationary phase (Fig. 1b vs. a). This trend is also true for the cgt $A$ (ts) mutant. At $30^{\circ} \mathrm{C}$, DNA synthesis continues to increase over the 2-h experiment, albeit at a 


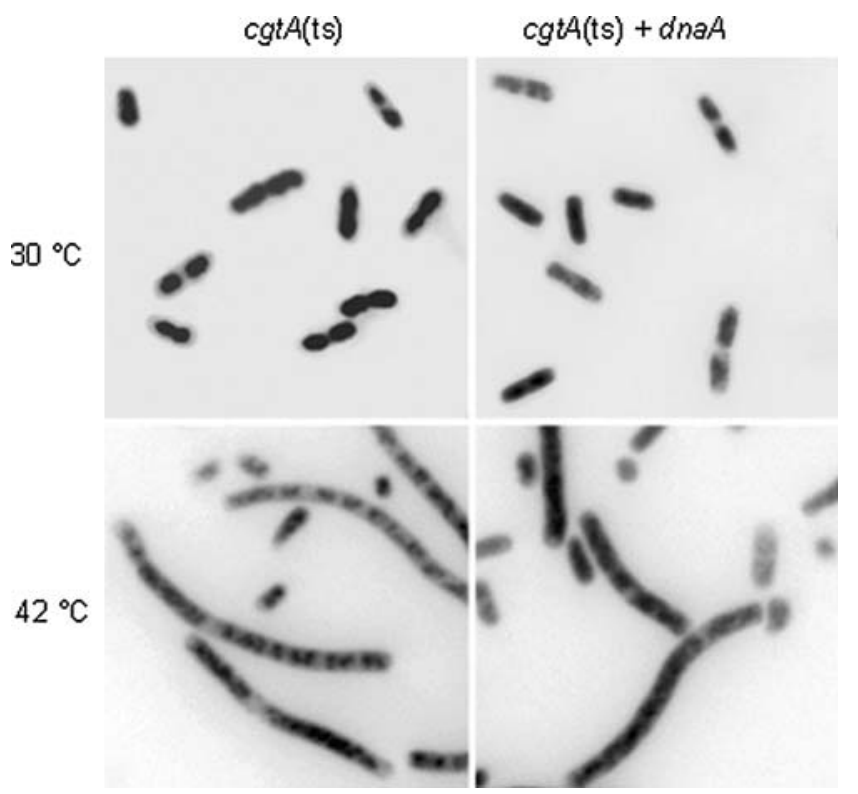

Fig. 2 Ectopic expression of dnaA does not suppress the cell division defect of $\operatorname{cgtA}(\mathrm{ts})$ mutants. Shown are gray-scale reverse images of DAPI-stained $\operatorname{cgt} A(\mathrm{ts})$ mutant cells alone or harboring the pdnaA116 $(\operatorname{cgt} A(\mathrm{ts})+d n a A)$ plasmid at either $30^{\circ} \mathrm{C}$ or after a 6-h shift to $42^{\circ} \mathrm{C}$, as indicated

level much reduced, as compared with the wild-type cells. At $42^{\circ} \mathrm{C}$, however, DNA synthesis is significantly impaired. The addition of pdnaA116 to $\operatorname{cgt} A$ (ts) cells resulted in an increase in DNA synthesis at $42^{\circ} \mathrm{C}$ (Fig. 1b). The rate of DNA synthesis, although significantly higher than that seen in the cgtA(ts) mutant alone, was still lower than that of the wild-type cells. Therefore, addition of pdnaA116 partially suppressed the DNA synthesis defect of the cgtA(ts) mutant. Again, ectopic expression of $\operatorname{seq} A$ had no significant effects on DNA synthesis in the $\operatorname{cgt} A$ (ts) mutant (data not shown).

DnaA protein levels in cells expressing the dnaA gene from a plasmid

To examine whether the observed partial suppression was due to the restoration or an increase in the DnaA levels, we performed immunoblot analysis on wild-type and $\operatorname{cgt} A$ (ts) cells harboring pdnaA116 using anti-DnaA antibodies. As reported earlier (Ulanowska et al. 2003), in $\operatorname{cgt} A$ (ts) bacteria grown at the non-permissive temperature, the levels of DnaA were dramatically reduced (Fig. 3a), whereas growth at $42^{\circ} \mathrm{C}$ did not affect the level of DnaA in wild-type cells (Fig. 3b). Interestingly, a significant reduction in the level of DnaA in the cgtA(ts) mutant occurred only after $2 \mathrm{~h}$ at the non-permissive temperature. The cgtA(ts) mutant cells harboring pdnaA116 possess equivalent levels of DnaA at either the permissive or non-permissive temperature (Fig. 3c). Thus, ectopic expression of $d n a A$ restored the levels of DnaA protein in the $\operatorname{cgt} A(\mathrm{ts})$ mutant.
Impairment of dnaA gene transcription in the $\operatorname{cgt} A$ (ts) mutant

To identify the step of $d n a A$ gene expression, which is affected in the $\operatorname{cgt} t A(\mathrm{ts})$ mutant, we have constructed a gene fusion bearing the lac $Z$ reporter gene under the control of the promoter region of dnaA. Analogous fusion, but containing a promoter of the seq $A$ gene, was also constructed. We found that CgtA inactivation resulted in the impairment of activity of the fusion bearing the dnaA promoter region, but not that of the seq $A$ promoter region (Fig. 4). Impaired expression of the dnaA gene in CgtA-deficient cells was also demonstrated at the level of mRNA (Fig. 5).

The stringent response occurs, albeit at a reduced level, in the $\operatorname{cgt} A$ (ts) mutant

All the results presented indicate that the expression of the dnaA gene is impaired in the cgtA mutant, and a resultant deficiency in DnaA protein activity causes inhibition of chromosomal DNA replication initiation. Since CgtA protein was found to be able to interact with ppGpp (Buglino et al. 2002) and the SpoT protein (Wout et al. 2004), one might assume that the observed effects of CgtA on DnaA could be indirect, due to any changes in the stringent control in cgtA(ts) mutants. In fact, $E$. coli chromosome replication is impaired under conditions of the stringent response (Guzman et al. 1988; Herman and Wegrzyn 1995), and expression of the dnaA gene is inhibited by ppGpp (Chiaramello and Zyskind 1990). Therefore, we examined whether the cgtA(ts) mutant is capable of demonstrating the stringent response.

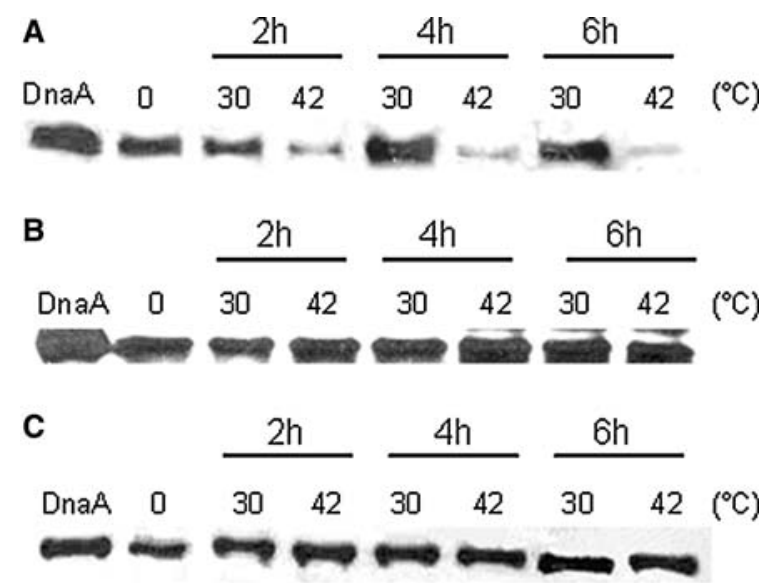

Fig. 3 The DnaA protein levels are restored in $\operatorname{cgt} A(\mathrm{ts})$-harboring pdnaA116. Cells were grown as described in Materials and methods and equivalent cell numbers, as judged by $\mathrm{OD}_{600}$, were removed prior to a temperature shift (labeled 0) or after temperature shift, as indicated $(2,4$ or $6 \mathrm{~h})$. The level of DnaA, as assayed by immunoblot analysis, is shown for $\operatorname{cgt} A(\mathrm{ts})$ (a), wildtype (b) and $\operatorname{cgt} A$ (ts)-harboring pdnaA116 (c) cells. Purified DnaA protein (labeled DnaA) was used as a standard 

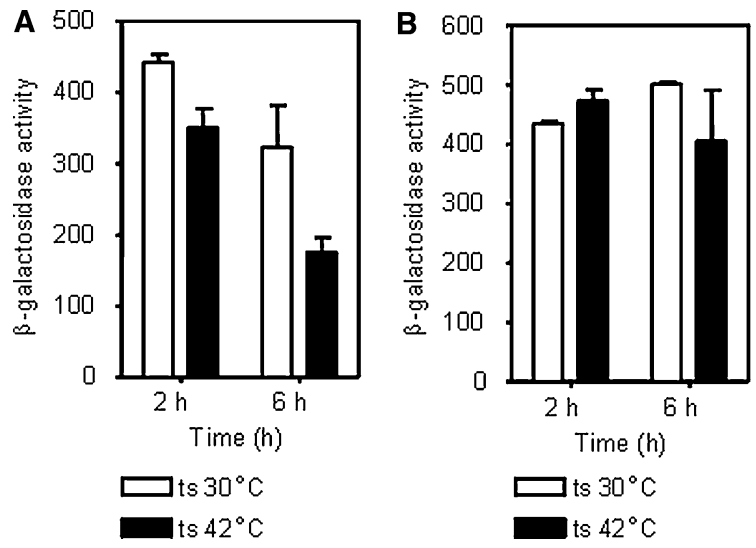

Fig. 4 Activities of the promoters of $\operatorname{dnaA}$ (a) and seqA (b) genes in $\operatorname{cgt} A$ (ts) cells, as estimated by the analysis of fusions of these promoters with the lac $Z$ reporter gene. Bacterial cultures were grown at $30^{\circ} \mathrm{C}$ to $\mathrm{OD}_{600}=0.05$. Then, a half of each culture was transferred to $42^{\circ} \mathrm{C}$ (closed columns) and the rest remained at $30^{\circ} \mathrm{C}$ (open columns). Culture samples were withdrawn after 2 and $6 \mathrm{~h}$ and $\beta$-galactosidase activities (in Miller units) were measured

We measured the level of total RNA synthesis based on the incorporation of $\left[{ }^{3} \mathrm{H}\right]$ uridine into TCA precipitable material. As noted earlier (Tosa and Pizer 1971), the addition of DL-serine hydroxamate induces the stringent response and results in a significant reduction in the level of stable RNA (i.e., rRNA and tRNA) synthesis (which corresponds to about $98 \%$ of transcriptional activity in bacterial cells; Fig. 6). In the cgt $A$ (ts) mutant, stable RNA synthesis occurs at low level at the nonpermissive temperature. However, the addition of DL-serine hydroxamate results in a further diminution of incorporation of radioactive uridine in cgtA(ts). Therefore, we conclude that E. coli cgtA mutants are capable of undergoing the stringent response. This phenotype could not be improved by the presence of pdnaA116.

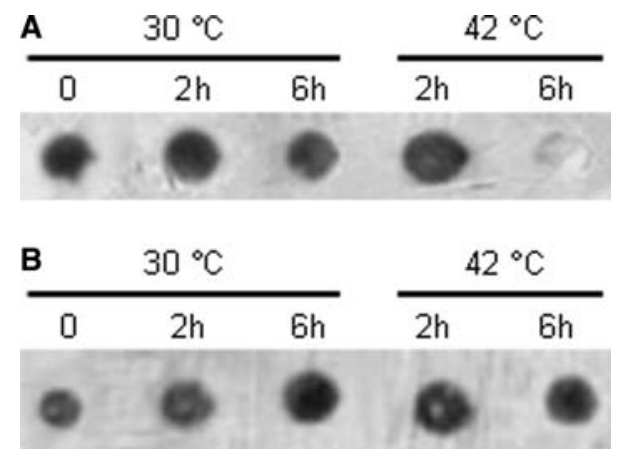

Fig. 5 Abundance of $\operatorname{dnaA}$ (a) and $\operatorname{seq} A$ (b) mRNAs in $\operatorname{cgt} A$ (ts) cells. Bacterial cultures were grown at $30^{\circ} \mathrm{C}$ to $\mathrm{OD}_{600}=0.05$ (labeled 0 on the figure). Then, a half of each culture was transferred to $42^{\circ} \mathrm{C}$ and the rest remained at $30^{\circ} \mathrm{C}$. Culture samples of the same bacterial mass (2 OD units) were withdrawn 2 and $6 \mathrm{~h}$ later and levels of mRNAs were estimated by hybridization with specific labeled probes

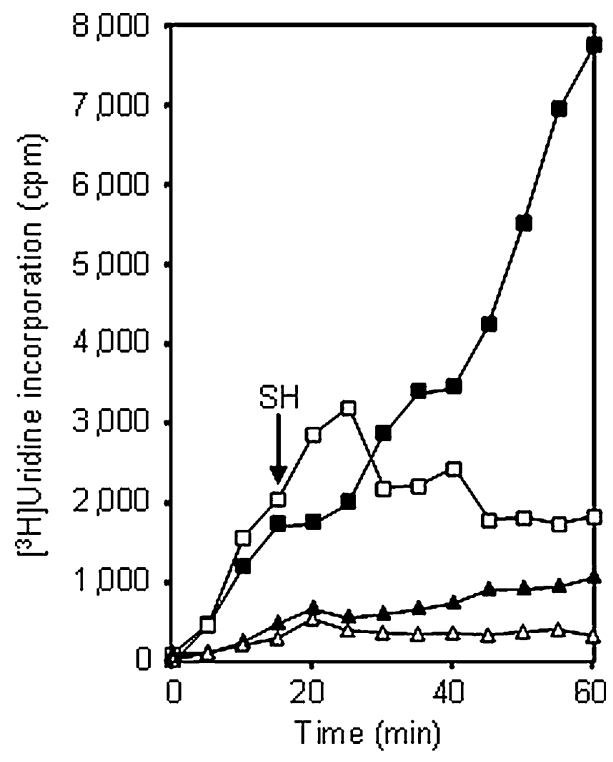

Fig. 6 Measurement of $\left[{ }^{3} \mathrm{H}\right]$ uridine incorporation under conditions resembling amino-acid starvation. Cells were grown as described in Materials and methods, except that, upon reaching an $\mathrm{OD}_{600}$ of 0.05 all cells were shifted to $42^{\circ} \mathrm{C}$. Upon reaching an $\mathrm{OD}_{600}$ of 0.2 (labeled as $0 \mathrm{~min}$ ), $\left[{ }^{3} \mathrm{H}\right]$ uridine was added. At the indicated time (arrow), DL-serine hydroxamate $\left(\mathrm{SH} ; 1 \mathrm{mg} \mathrm{ml}^{-1}\right.$ ) was added to induce the stringent response. Wild-type (squares) and cgtA(ts) mutant (triangles) with (open symbols) or without (closed symbols) the addition of DL-serine hydroxamate are shown

\section{Discussion}

Although cgtA gene product, the common GTP-binding protein (see Dutkiewicz et al. 2002 for discussion on the nomenclature), is an evolutionarily conserved protein and essential in E. coli (Arigoni et al. 1998), the crucial function(s) played by CgtA, which are necessary to support cellular growth, remains unclear. Since CgtA homologs in various bacterial species (B. subtilis, $C$. crescentus, E. coli) were shown to be associated with $50 \mathrm{~S}$ ribosomal subunit and interacted with GTP (Scott et al. 2000; Lin et al. 2004; Wout et al. 2004; Sato et al. 2005), one could assume that the lethal phenotype of cgtA dysfunction might be due to impaired ribosome function(s). However, studies on conditional C. crescentus cgtA G80E mutant, performed by Datta et al. (2004) revealed that this is not the case, and perhaps another process, crucial for cell viability, must be severely impaired when CgtA function is absent.

Here, we demonstrate that DNA replication is impaired in E. coli in the absence of CgtA function, indeed. It appears that CgtA regulates the expression of $d n a A$, a gene coding for the replication-initiator protein. Since ectopic expression of this gene results in partial restoration of both DNA synthesis and bacterial growth in the cgt $A$ mutant, one might speculate that the lethal effect of $c g t A$ dysfunction can be, at least partially, due to the depletion of DnaA. We could not, however, observe colonies of the $\operatorname{cgt} A(\mathrm{ts})$ mutant at $42^{\circ} \mathrm{C}$ even in the 
presence of a plasmid-bearing dnaA, and cell morphology was also not improved under these conditions.

Interestingly, in our experiments, we have used exactly the same strains as Kobayashi et al. (2001), who concluded that CgtA is not involved in DNA synthesis. However, they have performed flow cytometry analysis relatively shortly after the transfer of cells from permissive to nonpermissive temperature. As demonstrated by us in this report, significant effects on dnaA-expression level could be observed a few or several hours after such a transfer. Nevertheless, it should be noted that the $\operatorname{cgt} A$ (ts) mutant phenotype, which we observed is somewhat different from that reported previously for this strain (Kobayashi et al. 2001). For example, the growth defect was only apparent after $3 \mathrm{~h}$ at the nonpermissive temperature (Fig. 1a), as compared with $1 \mathrm{~h}$ reported earlier (Kobayashi et al. 2001). Since the strains are identical (which was confirmed by DNA sequencing), it is likely that these differences are due to various experimental approaches. In the earlier study, $0.1 \%$ arabinose was used for the induction of cgtA in both strains (Kobayashi et al. 2001). We have found, however, that this results in a vast overproduction of CgtA in GN5002 (data not shown), and therefore chose to induce each culture such that the level of CgtA was similar (data not shown). Also, cells in the earlier study were grown to stationary phase and then shifted directly to the nonpermissive temperature, whereas here we diluted overnight cultures and grew them at the permissive temperature for some time prior to a shift to the non-permissive temperature. The levels of CgtA are dramatically reduced in stationary-phase cells relative to exponentially growing cells (Sato et al. 2005), and therefore, the phenotypic differences could be due to a combination of the different levels of CgtA and the physiological state of the cells prior to the temperature shift.

The molecular mechanism of CgtA-mediated regulation of dnaA gene expression remains to be elucidated; in fact, serious effects of $c g t A$ dysfunction on the expression of another important gene, $\operatorname{rec} A$, have been reported recently (Zielke et al. 2003). The effects of $c g t A$ function on gene expression appear specific, as in control experiments we found that the ectopic expression of another gene coding for a replication-regulator protein (SeqA) was not affected in the cgt $A$ mutant. In the light of these results, a possibility that the CgtA dysfunction results in global inhibition of mRNA synthesis seems rather unlikely, despite the fact that we observed a marked reduction in stable RNA (rRNA and tRNA) synthesis (Fig. 6). We assume that this might be caused by impaired DNA replication and resultant inhibition of cell growth, which significantly influence stable RNA synthesis, rather than be a primary effect of $\operatorname{cgt} A$ mutation (note that bacterial cultures were incubated at the elevated temperature for a relatively long period before the onset of the measurement of RNA synthesis; see Materials and methods).

Kinetics of depletion of dnaA mRNA and DnaA protein after switching off the CgtA function may suggest that the regulation is on the levels of both tran- scription and translation. This latter hypothesis may be intriguing as the multiple roles of CgtA, for example, the regulation of DNA replication and translation remains an unsolved question. One might speculate that if CgtA senses the physiological state of the cell by responding to the GTP/GDP ratio, as a ribosome-associated protein, it may control the efficiency of ribosome biogenesis and translation of crucial regulatory genes, including dnaA. This control mechanism, enhanced by the regulation of dnaA transcription, might couple DNA replication to ribosome biogenesis and translation. Interestingly, it was proposed recently that yeast protein Yphlp might link cell proliferation control, DNA replication and ribosome biogenesis (Du and Stillman 2002). Finally, although we have demonstrated that the stringent response is normal in the cgtA mutant, one cannot exclude a possibility that interactions between CgtA and SpoT, demonstrated by Wout et al. (2004), influence the intracellular levels of ppGpp, thus affecting the expression of the dnaA gene, which was demonstrated earlier to be negatively regulated under conditions of the stringent response (Chiaramello and Zyskind 1990).

Acknowledgements We are grateful to Janine Maddock for fruitful discussions and continuous support during this study. This work was supported by National Institutes of Health (FIRCA program, project grant no. TW06001) and the Polish Ministry of Science and Informatics Technology (project grants no. 2 P04A 03027 to G.W. and 3 P04A 02922 to A.W.).

\section{References}

Arigoni F, Talabot F, Peitsch M, Edgerton MD, Meldrum E, Allet E, Fish R, Jamotte T, Curchod M-L, Loferer H (1998) A genome-based approach for the identification of essential bacterial genes. Nat Biotechnol 16:851-856

Buglino J, Shen V, Hakimian P, Lima CD (2002) Structural and biochemical analysis of the Obg GTP binding protein. Structure 10:1581-1592

Caldon CE, Yoong P, March PE (2001) Evolution of a molecular switch: universal bacterial GTPases regulate ribosome function. Mol Microbiol 41:289-297

Caldon CE, March PE (2003) Function of the universally conserved bacterial GTPases. Curr Opin Microbiol 6:135-139

Chiaramello AE, Zyskind JW (1990) Coupling of DNA replication to growth rate in Escherichia coli: a possible role for guanosine tetraphosphate. J Bacteriol 172:2013-2019

Czyż A, Weggrzyn G (2005) The Obg subfamily of bacterial GTPbinding proteins: essential proteins of largely unknown functions that are evolutionarily conserved from bacteria to humans. Acta Biochim Pol 52:35-43

Datta K, Skidmore JM, Pu K, Maddock JR (2004) The Caulobacter crescentus GTPase CgtA $_{C}$ is required for progression through the cell cycle and for maintaining 50S ribosomal subunit levels. Mol Microbiol 54:1379-1392

Du YC, Stillman B (2002) Yph1p, an ORC-interacting protein: potential links between cell proliferation control, DNA replication, and ribosome biogenesis. Cell 109:835-848

Dutkiewicz R, Słomińska M, Wegrzyn G, Czyż A (2002) Overexpression of the cgtA $(y h b Z, o b g E)$ gene, coding for an essential GTP-binding protein, impairs the regulation of chromosomal functions in Escherichia coli. Curr Microbiol 45:440-445

Foti JJ, Schienda J, Sutera VA Jr, Lovett ST (2005) A bacterial G protein-mediated response to replication arrest. Mol Cell 17:549-560 
Fuller RS, Funnell BE, Kornberg A (1984) The dnaA protein complex with the $E$. coli chromosomal replication origin (oriC) and other DNA sites. Cell 38:889-900

Giladi H, Igarashi K, Ishihama A, Oppenheim AB (1992) Stimulation of the phage lambda $\mathrm{pL}$ promoter by integration host factor requires the carboxy terminus of the alpha-subunit of RNA polymerase. J Mol Biol 227:985-990

Guzman EC, Carrillo FJ, Jimenez-Sanchez A (1988) Differential inhibition of the initiation of DNA replication in stringent and relaxed strains of Escherichia coli. Genet Res 51:173-177

Herman A, Węgrzyn G (1995) Effect of increased ppGpp concentration on DNA replication of different replicons in Escherichia coli. J Basic Microbiol 35:33-39

Kobayashi G, Moriya S, Wada C (2001) Deficiency of essential GTP-binding protein ObgE in Escherichia coli inhibits chromosome partition. Mol Microbiol 41:1037-1051

Kok J, Trach KA, Hoch JA (1994) Effects on Bacillus subtilis of a conditional lethal mutation in the essential GTP-binding protein Obg. J Bacteriol 176:7155-7160

Krause M, Ruckert B, Lurz R, Messer W (1997) Complexes at the replication origin of Bacillus subtilis with homologous and heterologous DnaA protein. J Mol Biol 274:365-380

Kukimoto-Niino M, Murayama K, Inoue M, Terada T, Tame JR, Kuramitsu S, Shirouzu M, Yokoyama S (2004) Crystal structure of the GTP-binding protein Obg from Thermus thermophilus HB8. J Mol Biol 337:761-770

Leipe DD, Wolf YI, Koonin EV, Aravind L (2002) Classification and evolution of P-loop GTPases and related ATPases. J Mol Biol 317:41-72

Lin B, Thayer DA, Maddock JR (2004) The Caulobacter crescentus $\mathrm{CgtA}_{C}$ protein cosediments with the free $50 \mathrm{~S}$ ribosomal subunit. J Bacteriol 186:481-489

Maddock J, Bhatt A, Koch M, Skidmore J (1997) Identification of an essential Caulobacter crescentus gene encoding a member of the Obg family of GTP-binding proteins. J Bacteriol 179:6426-6431

Messer W, Weigel C (2003) DnaA as a transcription regulator. Methods Enzymol 370:338-349

Michel B (2005) $\mathrm{Obg} / \mathrm{Ctg} \mathrm{A}$, a signaling protein that controls replication, translation, and morphological development? Dev Cell 8:300-301

Miller J (1972) Experiments in molecular genetics. Cold Spring Harbor Laboratory Press, Cold Spring Harbor
Okamoto S, Ochi K (1998) An essential GTP-binding protein functions as a regulator for differentiation in Streptomyces coelicolor. Mol Microbiol 30:107-119

Okamoto S, Itoh M Ochi K (1997) Molecular cloning and characterization of the obg gene of Streptomyces griseus in relation to the onset of morphological differentiation. J Bacteriol 179:170-179

Park JH, Jensen BC, Kifer CT, Parsons M (2001) A novel nucleolar G-protein conserved in eukaryotes. J Cell Sci 114:173-185

Sambrook J, Fritsch EF, Maniatis T (1989) Molecular cloning: a laboratory manual, 2nd edn. Cold Spring Harbor Laboratory Press, Cold Spring Harbor

Sato A, Kobayashi G, Hayashi H, Yoshida H, Wada A, Maeda M, Hiraga S, Takeyasu K, Wada C (2005) The GTP binding protein Obg homolog ObgE is involved in ribosome maturation. Genes Cells 10:393-408

Scott JM, Ju J, Mitchell T, Haldenwang WG (2000) The Bacillus subtilis GTP binding protein obg and regulators of the $\sigma^{\mathrm{B}}$ stress response transcription factor cofractionate with ribosomes. J Bacteriol 182:2771-2777

Tosa T, Pizer LI (1971) Biochemical bases for the antimetabolite action of L-serine hydroxamate. J Bacteriol 106:972-982

Trach K, Hoch JA (1989) The Bacillus subtilis spo0B stage 0 sporulation operon encodes an essential GTP-binding protein. J Bacteriol 171:1362-1371

Ulanowska K, Sikora A, Węgrzyn G, Czyż A (2003) Role of the $\operatorname{cgt} A$ gene function in DNA replication of extrachromosomal elements in Escherichia coli. Plasmid 50:45-52

Vidwans SJ, Ireton K, Grossman AD (1995) Possible role for the essential GTP-binding protein Obg in regulating the initiation of sporulation in Bacillus subtilis. J Bacteriol 177:3308-3311

von Freiesleben U, Krekling MA, Hansen FG, Lobner-Olesen A (2000) The eclipse period of Escherichia coli. EMBO J 19:62406248

Wout P, Pu K, Sullivan SM, Reese V, Zhou S, Lin B, Maddock JR (2004) The Escherichia coli GTPase CgtA $\mathrm{E}_{\mathrm{E}}$ cofractionates with the $50 \mathrm{~S}$ ribosomal subunit and interacts with SpoT, a ppGpp synthetase/hydrolase. J Bacteriol 186:5249-5257

Zielke R, Sikora A, Dutkiewicz R, Węgrzyn G, Czyż A (2003) Involvement of the cgtA gene function in stimulation of DNA repair in Escherichia coli and Vibrio harveyi. Microbiology 149:1763-1770 